\title{
Sequential learning and rule abstraction in Bengalese finches
}

\author{
Yumiko Yamazaki $\cdot$ Kenta Suzuki • Masayuki Inada • \\ Atsushi Iriki $\cdot$ Kazuo Okanoya
}

Received: 11 March 2011/Revised: 28 July 2011 / Accepted: 31 August 2011/Published online: 28 September 2011

(C) The Author(s) 2011. This article is published with open access at Springerlink.com

\begin{abstract}
The Bengalese finch (Lonchura striata var. domestica) is a species of songbird. Males sing courtship songs with complex note-to-note transition rules, while females discriminate these songs when choosing their mate. The present study uses serial reaction time (RT) to examine the characteristics of the Bengalese finches' sequential behaviours beyond song production. The birds were trained to produce the sequence with an "A-B-A" structure. After the RT to each key position was determined to be stable, we tested the acquisition of the trained sequential response by presenting novel and random threeterm sequences (random test). We also examined whether they could abstract the embedded rule in the trained sequence and apply it to the novel test sequence (abstract
\end{abstract}

Y. Yamazaki $(\bowtie) \cdot$ M. Inada $\cdot$ A. Iriki

Laboratory for Symbolic Cognitive Development,

RIKEN Brain Science Institute, 2-1 Hirosawa, Wako-shi,

Saitama 351-0198, Japan

e-mail: yumyam@brain.riken.jp

Y. Yamazaki

The Graduate School of Human Relations, Keio University, Mita Toho Building 2F, 3-1-7 Mita, Minato-ku,

Tokyo 160-8582, Japan

K. Suzuki · M. Inada - K. Okanoya

Laboratory for Biolinguistics, RIKEN Brain Science Institute,

2-1 Hirosawa, Wako-shi, Saitama 351-0198, Japan

K. Suzuki

Graduate School of Science and Engineering, Saitama

University, 255 Shimo-Okubo, Sakura-ku, Saitama-shi,

Saitama 338-8570, Japan

K. Okanoya

Department of Cognitive Behavioral Science,

Graduate School of Arts and Sciences, The University of Tokyo,

3-8-1 Komaba, Meguro-ku, Tokyo 153-8902, Japan test). Additionally, we examined rule abstraction through example training by increasing the number of examples in baseline training from 1 to 5 . When considered as (gender) groups, training with 5 examples resulted in no statistically significant differences in the abstract tests, while statistically significant differences were observed in the random tests, suggesting that the male birds learned the trained sequences and transferred the abstract structure they had learned during the training trials. Individual data indicated that males, as opposed to females, were likely to learn the motor pattern of the sequence. The results are consistent with observations that males learn to produce songs with complex sequential rules, whereas females do not.

Keywords Bengalese finch - Sequential learning . Serial reaction time task $\cdot$ Songbird

\section{Introduction}

Sequencing or ordering of behaviour has an important role in the effective survival of many animals. In a fixed action pattern (FAP), for example, the behavioural sequences, consisting of small elements of bodily movement, have meaning when considered as a whole. The specific placement of each element in the sequence is essential to the sequence's function (consider, for example, the "syntactic chain" in rodent grooming behaviour, Aldridge and Berridge 1998; Berridge et al. 1987).

Birdsong is another example of a sequential response governed by rules. Bengalese finches are known to produce complex songs, the structures of which contain different levels of integration of their parts, in which chunks contain several elements and phrases contain several chunks (Honda and Okanoya 1999; Seki et al. 2008). These parts 
of the song are organised by a rule that can be expressed as a finite-state syntax (Okanoya 2004). The functions of such complex songs are related to successful reproduction, because females are more likely to carry strings to prepare a nest when they hear complex songs than when they hear simple ones (Okanoya 2004). Thus, male Bengalese finches produce auditory sequences (i.e. songs) by unique rules governing the integration of small units (Okanoya 2002). Female Bengalese finches, on the other hand, appear to differentiate the songs on the basis of the integrative rules embedded in the songs as well as perceptual differences unique to each individual song.

The above facts have led us to address the following question: if the ability for processing sequential events is dependent on the ethological and neural characteristics of each sex in Bengalese finches, are different patterns of sequential ability demonstrated in cases where there are no songs? In the present study, we explored the ability of Bengalese finches to exhibit sequential behaviour by training them with arbitrary sequences of key-peck responses. We focused on each bird's ability to learn arbitrary sequential responses and to abstract the general structure embedded in the training sequences. Although the properties and mechanisms involved in Bengalese finch songs have been studied extensively, it has yet to be determined whether the sequential abilities expressed in the songs are independent of other types of sequential behaviour. We employed a "serial reaction time" (SRT) task to evaluate the learning and transfer of sequential responses.

The SRT task was developed to evaluate procedural memory function both in normal human subjects (Willingham et al. 1989) and in patients with neurological diseases (e.g. Knopman and Nissen 1991; Jackson et al. 1995). Recently, the SRT task has been used to study rats (Christie and Dalrymple-Alford 2004) and mice (Christie and Hersch 2004) using a modified version of the original task designed for humans (Schwarting 2009). In this model, subjects are trained to make serial responses to 4-12 stimuli that are presented in a specific order. The level of learning is indicated by the decrease in reaction time (RT) for the serial-ordering condition. Subsequently, they are tested with random sequences to determine whether they increase the response time in the random-sequence condition compared with the trained condition. In cured serially ordered conditions used with nonhuman animals, because the correct response positions are always cued by, for example, the brief illumination of a visual stimulus located at the response position, the task is much easier to acquire than one that employs the "simultaneous chaining" procedure, in which subjects have to learn to produce all sequences containing three to seven different responses without cueing (see Terrace 2005, for a review).

We used the SRT task to examine the serial learning ability of Bengalese finches. First, we determined whether
Bengalese finches could learn three-term sequential behaviour using key-peck responses. The birds were trained to peck successively at three different keys, guided by illumination of the keys. The SRT response for each response position was used as an index of the acquisition of sequential behaviour. If the birds had learned the trained sequence, then the SRT should decrease because the finches predict and prepare to peck at each response position. After they had successfully learned the three-term sequence, we used two different conditions to test what and how they learned in the baseline training. First, we used the random condition to test whether they had really learned the trained sequence. If the training conditions involved a sequence adhering to an embedded rule, the birds could abstract that embedded rule in the trained sequences and apply it to untrained ones. Thus, abstraction of the embedded rule, the second condition, was evaluated by presenting novel sequences that adhered to the same embedded rule to determine whether the birds' performed with the novel sequences as well as they did with the trained sequences. For example, in sequences such as 2-42 and 4-2-4, the first and third response positions are identical, so there are two possible ways to learn these sequence types: (1) absolute position learning, in which the conditions are exactly the same as those of the arbitrary sequence, and (2) abstract rule learning, in which the first and third positions are the same. If the abstract rather than the absolute rule were learned, the birds should be able to predict the position of the third response in novel test sequences containing the same response rule as the trained sequence (e.g. 5-1-5 and 3-2-3), which would result in little or no increase in RT for the third response position.

\section{Experiment}

We examined whether Bengalese finches could master arbitrary sequential responses using food-rewarded key pecks. To evaluate acquisition of the sequential response, we used SRT as an index. After being trained with a sequence containing the rule (e.g. 5-2-5, where the first and third response positions were identical), the birds were tested with novel sequences having the same rule (e.g. 3-4-3 and 2-3-2), which were referred to as "abstract tests", or with novel sequences having no rule (e.g. 2-4-3 and 4-2-5), which were referred to as "random tests", to see whether they based their response on the absolute positions learned during training or on the sequential rule abstracted from the trained example. If the birds used the abstract rule rather than absolute position, they should be able to predict the position of the third response in novel test sequences containing the same response rule as the trained sequence, which would result in little increase in $\mathrm{RT}$ for the third response position. 
In addition, we examined the effect of the number of training examples on test performance by comparing the results from training methods with different numbers of examples ( 1 vs. 5) to see whether the number of examples had any effect on abstraction learning (of the embedded rule).

\section{Methods}

\section{Subjects}

Twelve adult Bengalese finches (Lonchura striata var. domestica), six males and six females, were used in the experiment. Half of them were used in preliminary experiments for this study; thus, they could respond sequentially to the keys. The other half was experimentally naive. All birds were born in different families in our laboratory and were housed as a group. During the experimental period, they were kept at approximately $80 \%$ of their free-feeding weight. They had access to food in their cage for $2 \mathrm{~h}$ after each daily experimental session. Vitamin-enhanced water was freely available in their home cage. The light/dark cycle was set at $13 / 11 \mathrm{~h}$.

\section{Apparatus and stimuli}

Two operant boxes $(\mathrm{W} 15.5 \mathrm{~cm} \times \mathrm{D} 30.3 \mathrm{~cm} \times \mathrm{H} 22.0 \mathrm{~cm}$ ), specially arranged for Bengalese finches, were placed inside a soundproof box (W89 $\mathrm{cm} \times \mathrm{D} 70 \mathrm{~cm} \times \mathrm{H} 74 \mathrm{~cm}$, Music Cabin). On the front panel of the box (see Fig. 1), five response keys (transparent acrylic, $15 \mathrm{~mm}$ in diameter) were attached and aligned horizontally with $2.5 \mathrm{~cm}$ from centre to centre. The birds could peck at the key from the front perch. In addition, two small keys (the trial initiation (TI) keys, $10 \mathrm{~mm}$ in diameter) were installed under the five response keys, which were horizontally aligned on the centre and spaced $2.5 \mathrm{~cm}$ apart (as measured from the centres of each small key, see Fig. 1). The left key was illuminated with a green light, whereas the right key was illuminated with a red light. They were used as TI responses to start the sequential response task. An aperture $(3 \mathrm{~cm} \times 3 \mathrm{~cm})$, centred and placed $10 \mathrm{~cm}$ from the bottom of the panel, was used to obtain a food reward (a few grains of millet) after completing a sequence. When the feeder (Okubo Sokkoki) was operated and the food delivered, a small light illuminated the inside of the aperture. The pecking response was guided by the presentation of a white circle on the liquid crystal monitor (EIZO FlexScan L367, Nanao), which was observable through the transparent response keys. The house light remained on during the experimental session except for the period of reinforcement, during which only the feeder light was on.

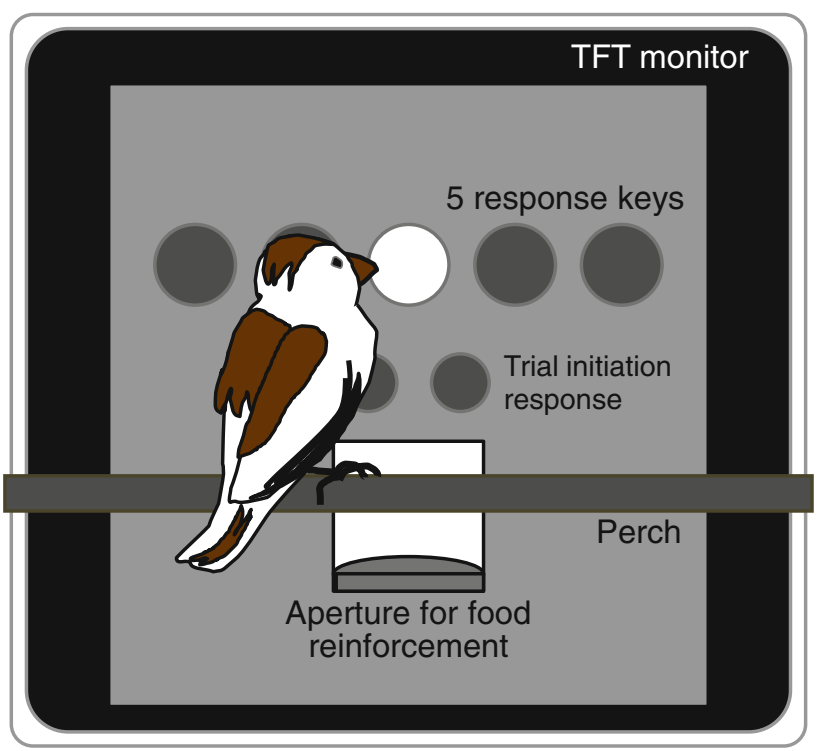

Fig. 1 Front panel of the testing apparatus used in the experiment. Five response keys were aligned horizontally, and an aperture for a food cup was placed below them. Two small keys were located below the five response keys for the trial initiation response. Behind the front panel, there was a TFT monitor for presentation of the white (five keys), and the red and green coloured circles (two trial initiation response keys)

\section{Procedure}

After being habituated to the feeder, the naive subjects were trained to peck each response key by reinforcing each response with a few grains of millet (presented by the feeder). Then the birds were trained to peck sequentially at the lit keys, which were randomly chosen, first with two and then with three different positions. After completing each sequence, the food reinforcement was delivered. Throughout this study, each daily session contained 60 trials. The average intertrial interval was $10 \mathrm{~s}$, with a range of $8-12 \mathrm{~s}$.

\section{Baseline training with one example}

After training the birds to randomly peck at the keys, all subjects were trained to peck at three keys sequentially, referred to as the baseline training in this experiment. Half of the subjects were assigned to respond sequentially to key positions 1-4-1, and the other half were assigned to respond to positions 5-2-5. One TI response was required to start the three-term sequential behaviour. After an intertrial interval, which was $10 \mathrm{~s}$ on average, either one of the TI keys was illuminated in green (left) or red (right). The position of the lit key was randomised so that the same position did not persist for more than three consecutive trials. After responding once to the illuminated TI key, the key light was extinguished and the first key position of the three-term sequential response was turned on. 
Each daily session contained 60 trials, and the probability of food reinforcement for each trial was set at 0.7. For trials when food reinforcement was not delivered after completion of the sequence (where the probability was 0.3 ), the light inside the food tray was lit for $4 \mathrm{~s}$ to provide a conditioned reinforcement. The birds were trained with one baseline sequence until the key-peck RT decreased and became stable. In this study, we defined the stability of performance during baseline training as having occurred when the standard deviations of the second and the third responses were both less than $1,000 \mathrm{~ms}$. Birds with a previous training history were trained for at least five sessions, whereas the naive subjects were trained for at least 20 sessions. The experimental tests were conducted after having confirmed that this criterion had been met for each subject.

\section{Random and abstract testing after training with one example}

After their performance during three-term sequence training had become completely stable, the birds were tested using the "random test". In this test, the RTs for each response position in the untrained sequences, randomly selected from a pool of 60 combinations, were measured. No repetition of the same position occurred in the random sequences (e.g. 3-3-5 and 1-2-2). The random test session consisted of 18 test trials intermixed with 42 baseline training trials. Food reinforcement was given after sequence completion in both the training and the test trials. It was expected that, once the trained sequence had been acquired fully by a Bengalese finch, the RT for untrained random sequences would increase.

In the "abstract test", 18 test trials were randomly interspersed with 42 training trials in a 60-trial test session. The testing sequences were selected from 20 possible sequences that had identical key positions for the first and third responses (e.g. 3-5-3 and 4-1-4). Regardless of whether the random and abstract test sessions involved a training or test trial, completion of the sequential response was reinforced by food presentation with a probability of 0.7. All birds experienced both test types, and the order was randomised for each sex.

\section{Baseline training with five examples}

Immediately after training and testing with one example, the birds were trained with five different rule-based sequences containing the same response position for the first and the third items (e.g. 1-3-1, 2-4-2, 3-5-3, 4-1-4, $5-1-5)$. We prepared eight sets, each containing five different sequences, which were assigned at random for each sex. Any of the key positions could be used as the starting position for each sequence. The birds were trained with these multiple sequences until their performance became stable (for at least 10 sessions).

\section{Random and abstract testing after training with five examples}

After their performance on three-term sequence training with five examples had become stable, the birds were retested using the same "random test" described above. The random test session contained 18 test trials intermixed with 42 baseline training trials. Reinforcement with a probability of 0.7 was given after completion of each training or test trial sequence.

The same "abstract test" was as used for training with one example. In this test, 18 test trials were randomly interspersed with 42 training trials in a 60-trial test session. Reinforcement was given after completion of each sequential response, as described above for the random test.

The order of the random and abstract tests was randomised for each sex. More than one training session was inserted between the tests to maintain stable baseline performance.

\section{Data analysis}

The RT for each response position in each three-term sequence was measured throughout this study. Any RTs greater than $10 \mathrm{~s}$ were excluded from further analysis. If the birds learned each response position in a trained sequence, the SRT on the random test trials would increase. We compared the RTs for the third item for the baseline and abstract test conditions because the RT for the third item in the abstract test was critical to evaluate the birds' ability of rule transfer. If the birds show the RTs which is not significantly different from the baseline in the abstract test trials but not in the random test trials, it would suggest that they transfer the specific rule trained in the baseline. Statistical significance was determined using two-tailed $t$ tests to compare group and individual performance data in the baseline and test trials.

For both sets of experimental conditions, two-way ANOVA was used to evaluate differences in baseline performances related to sex and experimental history.

\section{Results}

Baseline training with one example, and transfer tests

Two-way ANOVA (sex (male/female) $\times$ experimental experience (experienced/naive)) revealed no significant 
differences in baseline performances on the last day before the test session (sex: $F[1,8]=0.28, P=0.62$; experimental experience: $F[1,8]=0.003, P=0.95$; interaction: $F[1,8]=3.48, P=0.10)$. Thus, in the following tests, we did not separate the data for groups with different levels of experimental experience.

In the last baseline session before the random or abstract test series were performed, no statistically significant differences were observed between data obtained for males and females, as depicted in the left panel of Fig. 2 for every sequential position (the first response, $t(5)=0.090$, $P=0.932$; the second response, $t(5)=0.517, P=0.627$; the third response, $t(5)=0.141, P=0.893)$.

The RTs for each response position in the baseline and random test trials for males and females are depicted in Fig. 3. The RT data obtained for the third item was not significantly different, as determined by $t$ tests, between the baseline and test trials in male subjects $(t(5)=2.53$, $P=0.052)$. In females $(t(5)=0.66, P=0.535)$, this difference was also not significant.

The RTs for each response position in the baseline and abstract test trials for males and females are depicted in Fig. 3. In the abstract test, the differences between the baseline and test performance data obtained for the third item was insignificant for both males $(t(5)=2.35$, $P=0.065)$ and females $(t(5)=0.42, P=0.693)$.

Even if the results obtained demonstrate the birds' successful performance and preference for abstraction of the embedded rule (i.e. significant differences observed in the random test but not in the abstract test), the possibility remains that the birds were just learning the motor pattern of the ruled sequence. For example, in the group trained with the sequence " $1-4-1$ ", the subject might learn to move their body to the right after the first response and then to the left after the second response to obtain the food reinforcement. Thus, we separated the RTs in the test trials into learned or unlearned patterns of the motor sequence (i.e. right-left and left-right on the second and the third responses). When considered as groups, as shown, there were no significant differences between the learned and unlearned patterns for either sex (paired $t$ test: male: $t(5)=1.504, P=0.193$; female: $t(5)=0.034, P=$ $0.974)$. However, when analysed individually, we found that 4 out of 6 males and 1 out of 6 females showed significant difference between two categories of motor pattern (two sample $t$ test: bottom row of Table 1).

Baseline training with five examples, and transfer tests

Two-way ANOVA (sex (male/female) $\times$ experimental experience (experienced/naive)) revealed no significant differences in baseline performance on the last day before the test session (sex: $F[1,8]=0.44, P=0.52$; experimental experience: $F[1,8]=0.22, P=0.65$; interaction: $F[1,8]=0.0003, P=0.99)$. Thus, in the following tests, we did not conduct separate analyses of the data from groups with different levels of experience.

In the last baseline session before the random or abstract test series were performed, there was no statistically significant performance difference between males and females, as depicted in the right panel of Fig. 2 for every sequential position (the first response, $t(5)=0.334$, $P=0.752$; the second response, $t(5)=0.727, P=0.500$; the third response, $t(5)=0.476, P=0.654)$.

The average RTs recorded for the baseline trials and random test trials are depicted in Fig. 4. A significant difference in data obtained for these two trials was observed for males $(t(5)=4.99, P=0.004)$ but not for females $(t(5)=2.35, P=0.066)$.

The average RTs of the baseline trials and abstract test trials are depicted in Fig. 4. For performance related to the third item, differences between baseline and test performances were insignificant for both males $(t(5)=0.23$, $P=0.826)$ and females $(t(5)=2.37, P=0.063)$.

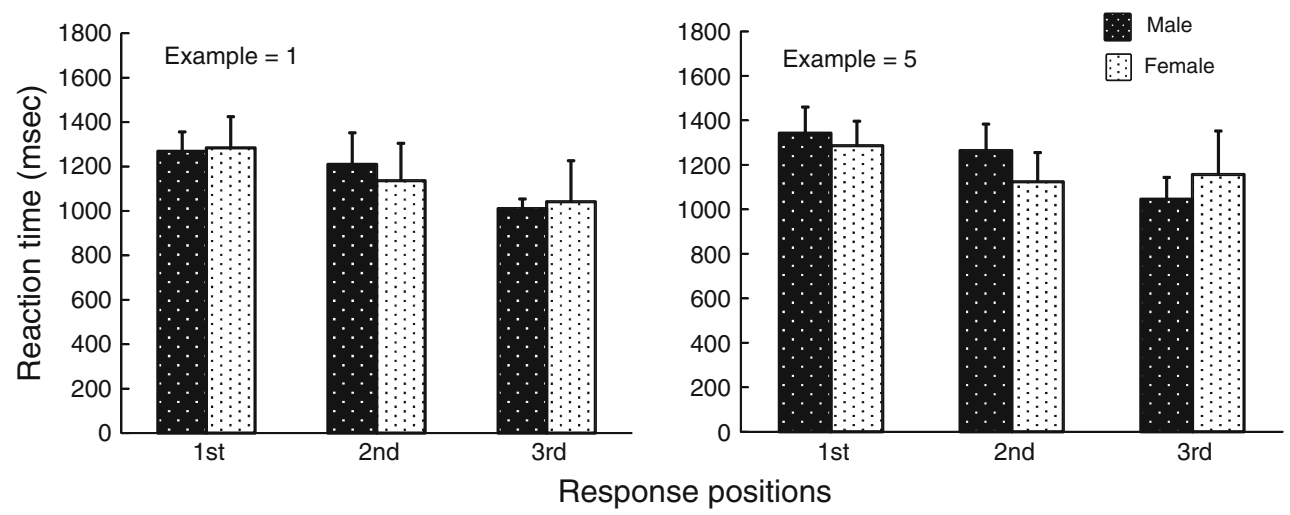

Fig. 2 RT for each response position of the sequence in each sex in the last session of baseline training with one (left panel) and five (right panel) examples, depicted with standard error of means 
Fig. 3 RT for each response position of the sequence in random (upper panels) and abstract (lower panels) tests after training with one example (depicted with standard error of means): males, left panel; females, right panel. The $P$ value is obtained from $t$ tests of the third response position between baseline and test trials
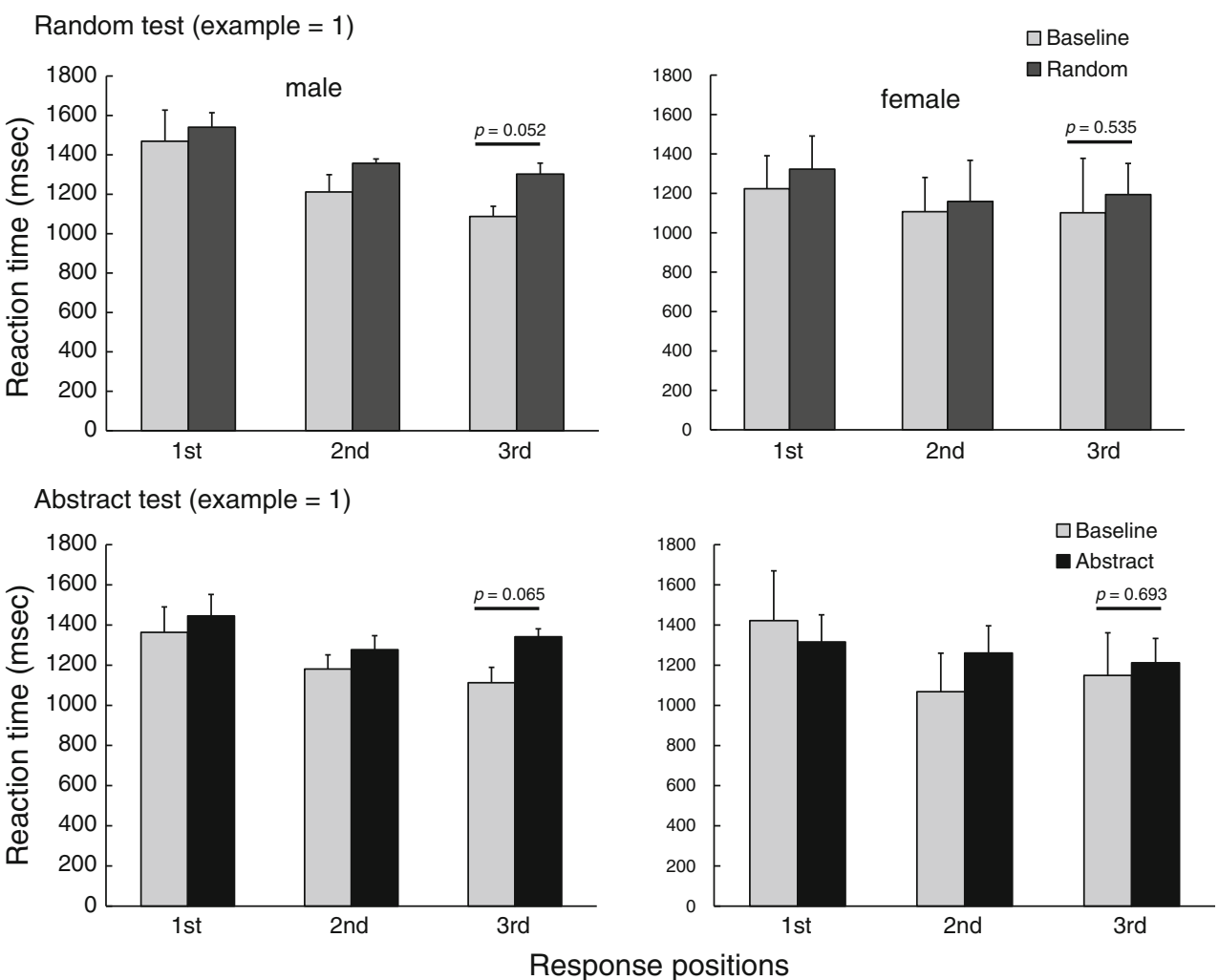

Table 1 Summary of the results of the $t$ tests of the third RTs between baseline and test trials in each bird, together with the group data for each sex and the results of motor learning

\begin{tabular}{|c|c|c|c|c|c|c|c|c|c|c|c|c|c|c|c|c|c|c|}
\hline \multirow[b]{2}{*}{ Subject } & \multicolumn{7}{|c|}{ Male } & \multicolumn{7}{|c|}{ Female } & \multicolumn{4}{|l|}{ Model } \\
\hline & 1 & 2 & 5 & 8 & 9 & 13 & Average & 3 & 4 & 6 & 10 & 11 & 12 & Average & Abstract & $\begin{array}{l}\text { Abstract } \\
\text { learning }\end{array}$ & $\begin{array}{l}\text { Sequence } \\
\text { adaptation }\end{array}$ & $\begin{array}{l}\text { Lack of } \\
\text { learning }\end{array}$ \\
\hline Random 1 & $* *$ & $* *$ & $*$ & NS & $*$ & NS & NS & $* *$ & NS & $* *$ & $* *$ & $* *$ & $* *$ & NS & $* *$ & $* *$ & $* * / N S$ & $* *$ \\
\hline Abstract 1 & $* *$ & NS & NS & NS & $* *$ & NS & NS & $*$ & NS & $* *$ & $* *$ & NS & NS & NS & NS & $* *$ & $* * / \mathrm{NS}$ & $* *$ \\
\hline Random 5 & $* *$ & $* *$ & NS & $* *$ & NS & $* *$ & $* *$ & NS & $* *$ & NS & NS & $*$ & $* *$ & NS & $* *$ & $* *$ & NS & $* *$ \\
\hline Abstract 5 & NS & NS & NS & NS & NS & $* *$ & NS & $* *$ & NS & NS & NS & $* *$ & NS & NS & NS & NS & NS & $* *$ \\
\hline Motor learning & $* *$ & $* *$ & $* *$ & NS & $*$ & NS & NS & NS & NS & NS & NS & NS & $*$ & NS & & & & \\
\hline
\end{tabular}

In case of analysis for motor learning, the RTs were compared between the test sequences with and without motor pattern consistent to the baseline sequence. Additionally, the right side of the table presents predicted results for 4 types of model birds, assuming (1) that the birds generalised the embedded rule after training with one example (abstract model), (2) that the birds generalised the embedded rule only after training with five examples (abstract learning model), (3) that the birds just adapted to the sequential responses after training with five examples (sequential adaptation model), and (4) that the birds learned nothing about the sequence even after training with five examples (lack-of-learning model)

$* P<0.05, * * P<0.01, N S$ no significant difference (two-tailed $t$ test)

To enable further inspection of individual performances during training and testing, Table 1 summarises the results of the $t$ tests between baseline and test trials for each bird, the group data obtained for each sex, and the motor learning results. Additionally, the right side of the table presents the results predicted for 4 types of bird models assuming (1) that the birds generalised the embedded rule after training with one example (abstract model), (2) that the birds generalised the embedded rule only after training with five examples (abstract learning model), (3) that the birds merely adapted to the sequential responses after training with five examples (sequential adaptation model), and (4) that the birds learned nothing about the sequence even after training with five examples (lack-of-learning model). The patterns of performance in subjects 2 (male) and 12 (female) are in accordance with those of the "abstract model", and the pattern of performance of subject 1 (male) is in agreement with the "abstract learning 
Fig. 4 RT for each response position of the sequence in random (upper panels) and abstract (lower panels) tests after training with five examples (depicted with standard error of means): males, left panel; females, right panel. The $P$ value is obtained from $t$ tests of the third response position between baseline and test trials
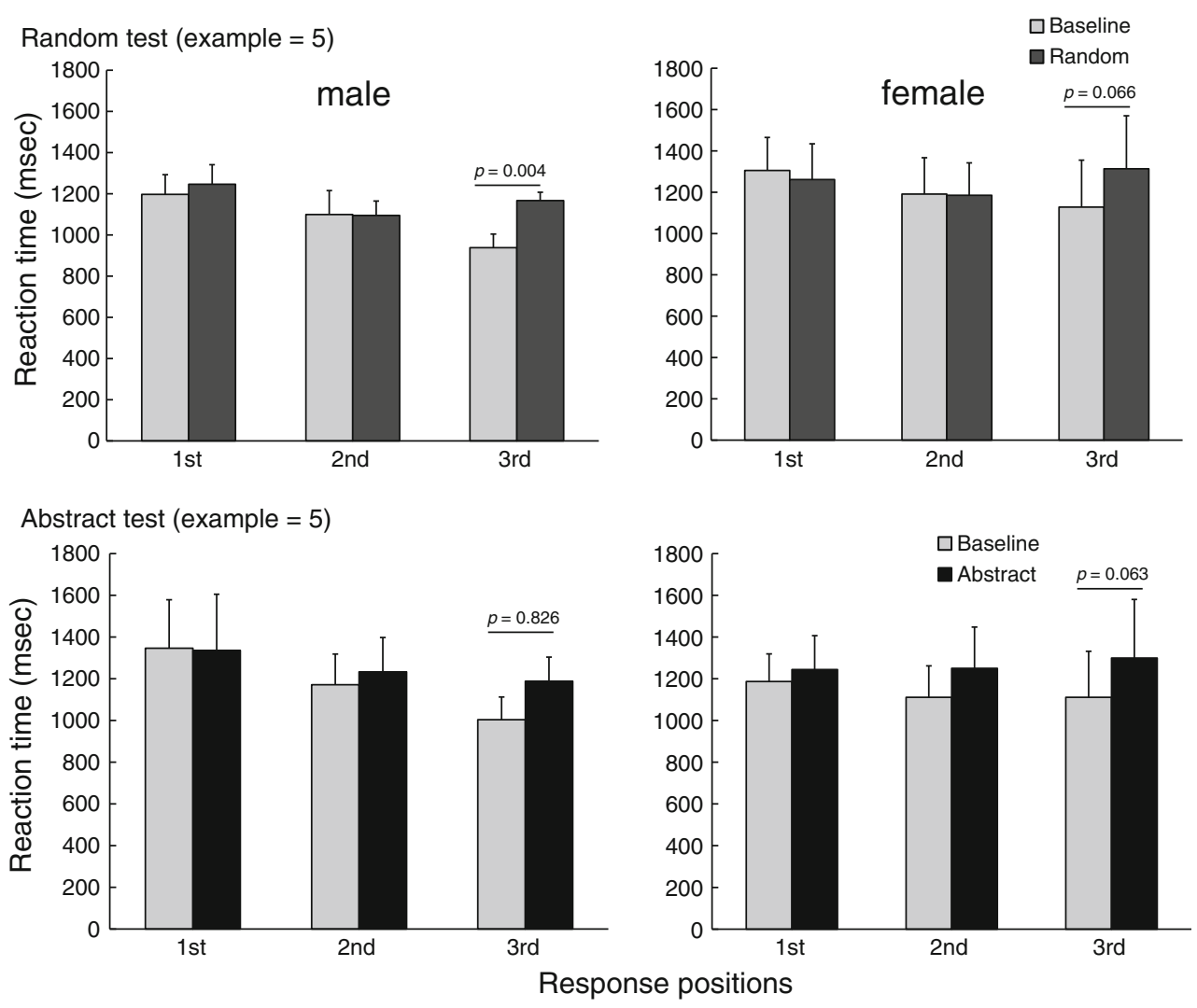

model". Consistent "sequential adaptation" performance was found in subjects 9 (male), 6, and 10 (female). There was no pattern of behaviour in either sex corresponding to the "lack-of-learning model". Thus, there were no substantial gender-related differences in the numbers of subjects exhibiting rule abstraction and sequence adaptation. However, 4 out of 6 male subjects exhibited motor learning, compared to only 1 out of 6 females. Note that all subjects whose performances were in accordance with the "abstract" or "abstract learning" models exhibited motor learning (subjects 1, 2, and 12).

\section{Discussion}

The results of the present study, using the SRT task model, show that Bengalese finches can acquire three-term sequential behaviour, as indicated by RT decreases. This is the first time that the SRT task model has been used with this species, and it proved to be effective for evaluating the learning of sequential behaviour. Learning proceeded quite quickly, and performance remained stable when the number of training examples was increased from 1 to 5 . For both random and abstract tests, the birds' performance was unchanged after training with one example. After being trained with five examples of the rule-based sequence, however, males showed significantly longer RTs on the third item in the random test trials, compared with the baseline training trials; no significant change was observed for females. On the other hand, in the abstract test, both the males and the females showed no significant difference in the third sequential RT obtained for the abstract test versus the baseline training trials. This indicates that the males learned each sequence exactly as it occurred during the training sets and transferred the abstract structure embedded within and shared between each sequence. The females' performance indicates that they could be easily trained to detect and quickly respond to the lit keys to obtain food reinforcement.

What was learned in the course of training?

Although the group data in the random and abstract tests after five examples support the males' ability to abstract embedded rules, it remains unclear why there was no significant difference between the test and baseline trials in either test after training with one example. If individuals were not transferring the sequence rule, it should follow that latencies would be longer for both the random and abstract test sequences (as observed for the "abstract learning model" in Table 1). Upon further inspection of the individual data, we found that both males and females could be categorised within the "abstract" or "abstract learning" groups (see Table 1). 
When we analysed the data on the basis of the motor pattern required in each test trial, there appears to be a strong tendency for motor learning in males trained with only one example, however. The individual results of the abstract test after one example indicated that they responded significantly faster when the motor pattern required in the test trials was consistent to that of the baseline trials. In this regard, the fact that the male subjects showing abstract learning after five examples all exhibited motor learning after one-example training suggests that they are able to achieve rule abstraction in the five-example training via motor learning in the one-example training. Thus, although the cognitive ability to abstract the embedded rule in arbitrary sequences does not appear to be differentiated, the manner of learning sequential rule might have been achieved differently between the sexes.

Additionally, the obtained results are quite interesting in the light of the neural circuits in this species. In songbirds, the anterior forebrain pathway (AFP) is a highly specialised pallial-basal ganglia circuit that is homologous to the cortical-basal ganglia circuit in mammals (Doupe et al. 2005). The AFP is crucial in song learning and song plasticity but not in song production (Bottjer et al. 1984; Scharff and Nottebohm 1991; Brainard and Doupe 2000). The hypothesis that AFP has a critical role in abstracting general structure from sequential events is consistent with findings of a bias in motor learning towards males rather than females, the latter of which do not have such a song circuit. It may be reasonable for female Bengalese finches to display sequential abilities not via a production method but via a recognition method that discriminates male songs based on their notes and complexity (Okanoya 2004). If this hypothesis was correct, the role of the AFP would be extended to learning general types of sequential behaviours in a manner similar to the function of the basal ganglia in mammals (Graybiel 2000, 2008).

Bengalese finches of both sexes can recognise songs. Males use online feedback loops from their own produced songs to monitor their song production (Sakata and Brainard 2006), and females become more actively involved in nest preparation when they hear songs containing complex constructions (Okanoya 2004). The production and perception of songs by males can be made consistent with the motor theory of speech perception (e.g. Liberman and Mattingly 1985; Galantucci et al. 2006) by assuming that the motor system involved in song production is also recruited for their perception. This theory seems to contradict evidence for song discrimination in female Bengalese finches, because they have no neural mechanisms for producing songs. However, the perceptual abilities of females suggest that they might abstract more than phonological elements from the songs, such as their complexity or their overall vigour, without requiring the ability to analyse their detailed content. Such information would have little relation to elements produced by motor responses, which may synchronise visual information such as dancing (Seki and Okanoya 2008). Thus, mechanisms of perception or levels of recognition of biologically important events might be modulated differently in each sex via neural connections that play a specialised role in reproduction.

Rule abstraction in humans and non-human animals

Humans, with their use of grammar for communication, are much more capable of finding embedded structure in a sequence than non-human animals. This ability may not be completely innate but can be acquired with considerable speed early in life, as has been found in young children well before they master language (Marcus et al. 1999).

Despite their lack of linguistic communication, this ability can also be observed in animals other than humans. Using Pavlovian conditioning, it has been shown recently that rats can use sequential visual or auditory information to extract the essential abstract structure of a sequence of auditory items (Murphy et al. 2008). For instance, they can discriminate between sequences with or without repetition of the first event (i.e. ABA or ABB). These studies suggest that non-human as well as human animals can recognise the rules embedded in sequential events. Another type of study has suggested that non-human animals are able to recognise sequential events by transferring trained sequential responses. Starlings exhibited the ability to discriminate events with or without a recursive structure, such as in phrase grammar (Gentner et al. 2006), although whether they really learned and transferred the recursive structure is still a subject of debate (cf., Corballis 2007). In addition, pigeons have been shown to discriminate between sequences with or without grammatical structure (Herbranson and Shimp 2008). On the basis of these results, it is possible that a broad range of species is capable of recognising the abstract structure of sequential events.

In contrast, when rhesus macaques were trained to perform ABA-like sequences, they did not show any indication of transferring the rules embedded within the trained sequences (Procyk et al. 2000). Most of the time, their responses were controlled by the absolute sequential order of the individual elements. Although complex sequences comprising six items were used, human subjects could clearly transfer abstract structure only if they had been instructed before the experiment that the sequences had some abstract, common structure (Dominey et al. 1998). Based on these results, there may appear to be no homological basis for rule-learning ability. However, this could be partially attributable to the methodologies employed by individual studies. In general, such studies evaluate the ability to recognise or discriminate between 
trained and novel structures in successful reports using Pavlovian conditioning and two-choice discrimination tasks. These can be categorised as "reception methods" that determine how the subjects understand the structure of a sequence. In contrast, Procyk et al. (2000) were unsuccessful when they employed a "production method" that required the animals to learn trained sequences and produce novel sequences by applying the abstracted structure. Although the latter may inflict a heavier cognitive and motor load on the subjects than the former, it is important to recognise the difference between the two methods, specifically that the cognitive aspects of both types of tasks are necessary conditions for using language to communicate among members of human linguistic communities. If nonhuman animals are able to recognise the rules embedded in sequences but are unable to apply the abstract rule when different items are used, then the transfer and application of an abstract rule would be an ability that is highly biased towards humans. Alternatively, if some evolutionary factor contributed to this ability, then species having behavioural characteristics similar to the structure of human language would show a special ability to transfer and apply abstract rules. Because Bengalese finches show evidence for such abilities, it is more plausible that common biological and ethological pressures for communication and mate attraction, shared by humans and this species, are critical in determining complex sequential ability.

Acknowledgments This study complies with the current laws of Japan (Act on Welfare and Management of Animals). All experimental procedures and handling methods were performed in accordance with the "Guidelines for Conducting Animal Experiments" of RIKEN. The experiments were approved by the Animal Experiment Committee at RIKEN. This study was supported by Grant-in-Aid for Young Scientists (B) (17730441) for the first author.

Open Access This article is distributed under the terms of the Creative Commons Attribution Noncommercial License which permits any noncommercial use, distribution, and reproduction in any medium, provided the original author(s) and source are credited.

\section{References}

Aldridge JW, Berridge KC (1998) Coding of serial order by neostriatal neurons: a 'natural action' approach to movement sequence. J Neurosci 18:2777-2787

Berridge KC, Fentress JC, Parr H (1987) Natural syntax rules control action sequence of rats. Behav Brain Res 23:59-68

Bottjer SW, Miesner EA, Arnold AP (1984) Forebrain lesions disrupt development but not maintenance of song in passerine birds. Science 224:901-903

Brainard MS, Doupe AJ (2000) Interruption of a basal gangliaforebrain circuit prevents plasticity of learned vocalizations. Nature 404:762-766

Christie MA, Dalrymple-Alford J (2004) A new rat model of the human serial reaction time task: contrasting effects of caudate and hippocampal lesions. J Neurosci 24:1034-1039
Christie MA, Hersch SM (2004) Demonstration of nondeclarative sequence learning in mice: development of an animal analog of the human serial reaction time task. Learn Mem 11:720-723

Corballis MC (2007) Recursion, language, and starlings. Cogn Sci 31:697-704

Dominey PF, Lelekov T, Ventre-Dominey J, Jeannerod M (1998) Dissociable processes for learning the surface structure and abstract structure of sensorimotor sequences. J Cogn Neurosci 10:734-751

Doupe AJ, Perkel DJ, Reiner A, Stern EA (2005) Birdbrains could teach basal ganglia research a new song. Trends Neurosci 28:353-363

Galantucci B, Fowler CA, Turvey MT (2006) The motor theory of speech perception reviewed. Psychon Bull Rev 13:361-377

Gentner TQ, Fenn KM, Margoliash D, Nusbaum HC (2006) Recursive syntactic pattern learning by songbirds. Nature 440:1204-1207

Graybiel AM (2000) The basal ganglia. Curr Biol 10:R509-R511

Graybiel AM (2008) Habits, rituals, and the evaluative brain. Ann Rev Neurosci 31:359-387

Herbranson WT, Shimp CP (2008) Artificial grammar learning in pigeons. Learn Behav 36:116-137

Honda E, Okanoya K (1999) Acoustical and syntactical comparisons between songs of the white-backed munia Lonchura striata and its domesticated strain, the Bengalese finch Lonchura striata var. domestica. Zool Sci 16:319-326

Jackson GM, Jackson SR, Harrison J, Henderson L, Kennard C (1995) Serial reaction time learning and Parkinson's disease: evidence for a procedural learning deficit. Neuropsychologia 33:577-593

Knopman D, Nissen MJ (1991) Procedural learning is impaired in Huntington's disease: evidence from the serial reaction time task. Neuropsychologia 29:245-254

Liberman AM, Mattingly IG (1985) The motor theory of speech perception revised. Cogn 21:1-36

Marcus GF, Vijayan S, Rao SB, Vishton PM (1999) Rule learning by seven-month-old infants. Science 283:77-80

Murphy RA, Mondragón E, Murphy VA (2008) Rule learning by rats. Science 319:1849-1851

Okanoya K (2002) Sexual display as a syntactical vehicle: the evolution of syntax in birdsong and human language through sexual selection. In: Wray A (ed) The transition to language. Oxford University Press, UK, pp 46-63

Okanoya K (2004) The Bengalese finch: a window on the behavioral neurobiology of birdsong syntax. Ann NY Acad Sci 1016:724-735

Procyk E, Dominey PF, Amiez C, Joseph J (2000) The effects of sequence structure and reward schedule on serial reaction time learning in the monkey. Cogn Brain Res 9:239-248

Sakata JT, Brainard MS (2006) Real-time contributions of auditory feedback to avian vocal motor control. J Neurosci 26:9619-9628

Scharff C, Nottebohm F (1991) A comparative study of the behavioral deficits following lesions of various parts of the zebra finch song system: implications for vocal learning. J Neurosci 11:2896-2913

Schwarting RK (2009) Rodent models of serial reaction time tasks and their implementation in neurobiological research. Behav Brain Res 199:76-88

Seki Y, Okanoya K (2008) Sex differences in audiovisual discrimination learning by bengalese finches (Lonchura striata var. domestica). J Comp Psychol 122:26-34

Seki Y, Suzuki K, Takahasi M, Okanoya K (2008) Song motor control organizes acoustic patterns on two levels in bengalese finches (Lonchura striata var. domestica). J Comp Physiol A 194:533-543

Terrace HS (2005) The simultaneous chain: a new approach to serial learning. Trends Cogn Sci 9:202-210

Willingham DB, Nissen MJ, Bullemer P (1989) On the development of procedural knowledge. J Exp Psychol Learn Mem Cogn 15:1047-1060 